Louisiana State University

LSU Digital Commons

Faculty Publications

Department of Chemistry

$4-19-2013$

\title{
Triphosgene-amine base promoted chlorination of unactivated aliphatic alcohols
}

Andrés Villalpando

Louisiana State University

Caitlan E. Ayala

Louisiana State University

Christopher B. Watson

Louisiana State University

Rendy Kartika

Louisiana State University

Follow this and additional works at: https://digitalcommons.Isu.edu/chemistry_pubs

\section{Recommended Citation}

Villalpando, A., Ayala, C., Watson, C., \& Kartika, R. (2013). Triphosgene-amine base promoted chlorination of unactivated aliphatic alcohols. Journal of Organic Chemistry, 78 (8), 3989-3996. https://doi.org/ $10.1021 /$ jo400341n

This Article is brought to you for free and open access by the Department of Chemistry at LSU Digital Commons. It has been accepted for inclusion in Faculty Publications by an authorized administrator of LSU Digital Commons.

For more information, please contact ir@lsu.edu. 


\title{
Triphosgene-Amine Base Promoted Chlorination of Unactivated Aliphatic Alcohols
}

\author{
Andrés Villalpando, Caitlan E. Ayala, Christopher B. Watson ${ }^{\dagger}$, and Rendy Kartika ${ }^{*}$ \\ Department of Chemistry, Louisiana State University, 232 Choppin Hall, Baton Rouge, Louisiana \\ 70803, United States
}

\section{Abstract}

Unactivated $\alpha$-branched primary and secondary aliphatic alcohols have been successfully transformed into their corresponding alkyl chlorides in high yields upon treatment with a mixture of triphosgene and pyridine in dichloromethane at reflux. These mild chlorination conditions are high yielding, stereospecific, and well tolerated by numerous sensitive functionalities. Furthermore, no nuisance waste products are generated in the course of the reactions.

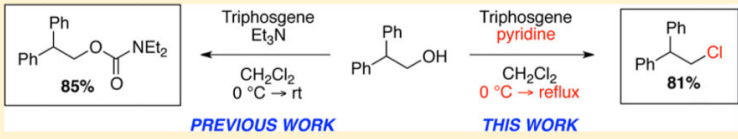

\section{INTRODUCTION}

The preparation of alkyl chlorides continues to be an area of significant interest in organic synthesis. While several new methods have been developed in recent years, many of these have limited variability of substrate tolerance and efficiency. ${ }^{1-6}$ Our involvement in the development of new chlorination methodology originated from our synthetic interests in chlorine-containing natural products, such as the chlorosulfolipids (Figure 1). The chlorosulfolipid class of natural products is essentially highlighted by multiple chlorine and sulfate sub-stitutions along the long hydrocarbon backbone. These note-worthy features are accompanied by multiple stereogenic centers of chlorine atoms, which present significant challenges for structure elucidation and total synthesis. ${ }^{7,8}$

Intrigued by the unusual structural features of this class of natural products, we initiated research to develop a mild method for chemoselective chlorination of aliphatic alcohols, particularly in the presence of nearby sensitive functionalities. In fact, we recently reported a new method for the chemoselective chlorination of primary aliphatic alcohols using a mixture of triphosgene and triethylamine in dichloromethane. ${ }^{9}$ As shown in Scheme 1, these conditions were effective in producing primary alkyl chlorides in high yields while being

(C) 2013 American Chemical Society

*Corresponding Author: R.K.: rkartika@1su.edu.. AUTHOR INFORMATION

The authors declare no competing financial interest.

$\dagger$ Undergraduate research participant. 
compatible with various acid- and base-sensitive functionalities that would be problematic under classical conditions. Furthermore, these conditions were operationally simple, due to the fact that triphosgene exists as a stable nonhygroscopic crystalline material at room temperature, and this permits easy and safe handling. ${ }^{10-12}$

During the course of substrate studies, we observed that sterically hindered alcohols were not suitable for our methodology. For example, activation of 2,2-diphenyl-1-ethanol (1) with a triphosgene-triethylamine mixture yielded only diethylcarbamate adduct $\mathbf{2}$ in $85 \%$ yield, while the chlorination product was not detected. Furthermore, exposure of secondary alcohol $\mathbf{3}$ under identical conditions produced a mixture of alkyl chloride $\mathbf{5}$ and diethylcarbamate $\mathbf{6}$ in $45 \%$ and $27 \%$ yields, respectively. It appeared that the selectivity between formation of alkyl chloride and diethylcarbamate was driven by sterics via an intermediacy of acylammonium ion species $\mathbf{7}$, where the ensuing nucleophilic attack by chloride ions could competitively occur at two possible electrophilic carbon centers. ${ }^{13-15}$.

Our mechanistic investigation also concluded that alcohols remain unreactive toward triphosgene in the absence of triethylamine, which serves as a base and nucleophilic catalyst to promote chlorination. ${ }^{9}$ The broader applicability of our method to sterically congested substrates is clearly limited by these selectivity problems, and therefore, it is imperative that we address these crucial issues.

\section{RESULTS AND DISCUSSION}

As shown in Table 1, we proceeded with a comprehensive optimization study by initially varying the amount of triethylamine while maintaining 0.5 equiv of triphosgene. Secondary alcohol $\mathbf{3}$ was used as a model substrate for these studies. We hypothesized that perhaps reducing the amount of triethylamine would inhibit formation of the undesired diethylcarbamate functionality. As shown in entry 1 , the use of 1.0 equiv of triethylamine predominantly produced chloroformate $\mathbf{4}$ along with minimal alkyl chloride $\mathbf{5}$ and diethylcarbamate $6 .{ }^{16}$ According to our expectation, an incremental increase of triethylamine from 1.0 to 2.0 equiv resulted in the disappearance of 4 . In these cases, however, formation of $\mathbf{5}$ and $\mathbf{6}$ was found to be equally facile. As indicated in entries 2-5, the two competing products were produced in a nearly 1:1 ratio regardless of the quantity of triethylamine. Interestingly, entries 6-10 indicated the resistance of chloroformate 4 to further transformation to either products 5 or $\mathbf{6}$ when an identical series of optimization reactions were executed in toluene.

As a result of these initial studies, we concluded that triethylamine alone, as a base/activator, would not be effective for our intended chlorination. Optimization studies then shifted to the exploration of a mixture of amine bases, such as pyridine and triethylamine. On the basis of the proposed $\mathrm{N}$-acylammonium ion intermediate $7,{ }^{9}$ our chlorination reaction could employ a stoichiometric amount of pyridine, which would serve as a base in the chloroformylation step, ${ }^{16}$ while maintaining a substoichiometric amount of triethylamine to promote chlorination. We realized that formation of triethylammonium ion species would be favored in the proton transfer equilibrium between protonated pyridine and triethylamine upon chloroformylation of the alcohol starting material, due to the difference in the $\mathrm{p} K_{\mathrm{a}}$ values of 
their conjugate acids. ${ }^{17}$ However, a low concentration of unprotonated triethylamine is expected to remain in the equilibrium and should promote nucleophilic substitution by chloride ions by forming acylammonium ion intermediate 7 .

As shown in Table 2, entries 1-8, activation of secondary alcohol 3 with triphosgene in the presence of 1.2 equiv of pyridine with varying substoichiometric amounts of triethylamine successfully suppressed the formation of diethylcarbamate $\mathbf{6}$. These reactions primarily afforded chloroformate 4 at room temperature (entries 1-4), but heating the reaction mixtures to reflux (entries 5-8) led to an increased production of alkyl chloride 5. In fact, entry 7 details the GC-MS analysis of the crude materials, revealing that the use of 1.2 equiv of pyridine and 0.75 equiv of triethylamine in dichloromethane at reflux quantitatively converted secondary alcohol 3 to alkyl chloride $\mathbf{5}$. Much to our surprise, an attempt to further investigate the role of pyridine in this reaction yielded unanticipated results, as studies in entries 9-12 indicated that the use of pyridine by itself readily generated the target alkyl chloride. In fact, secondary alcohol $\mathbf{3}$ was completely consumed and converted to alkyl chloride 5 when the reaction was performed using excess pyridine in refluxing dichloromethane (entries 13-16). These conditions completely eliminated the problematic formation of diethylcarbamate byproduct. Interestingly, while the use of 1.0 equiv of pyridine led to a mixture of 2:1 mixture of starting material $\mathbf{3}$ and alkyl chloride $\mathbf{5}$ (entry 13), increasing the amount of pyridine to 1.2 equiv fully consumed alcohol $\mathbf{3}$ and yielded a 1:1 mixture of chloroformate $\mathbf{4}$ and alkyl chloride 5 (entry 14). These observations again strongly suggested an intermediacy of the chloroformate species in our chlorination reaction.

These results were intriguing, as there are precedents which demonstrate that a triphosgenepyridine mixture readily chlorinates activated alcohols, such as those in benzylic, allylic, or propargylic systems. ${ }^{18}$ There are also reports that demonstrate the use of this mixture to convert aliphatic alcohols to their corresponding chloroformates. ${ }^{19-22}$ However, to the best of our knowledge, chlorination of unreactive aliphatic secondary alcohols using this mixture has remained unexplored. With these preliminary results in hand, we then examined the generality of these chlorination conditions by screening a series of secondary alcohols containing various common functional and protecting groups. A typical reaction protocol involved addition of 0.5 equiv of triphosgene and 2.0 equiv of pyridine to a solution of 1.0 equiv of secondary alcohol in dichloromethane at $0{ }^{\circ} \mathrm{C}$. The reaction mixture was then warmed to gentle reflux overnight, followed by aqueous workup with a dilute $\mathrm{HCl}$ solution and flash chromatography. It is crucial to note that unlike the classical chlorination methods using $\mathrm{SOCl}_{2}$ or $\mathrm{PPh}_{3}-\mathrm{NCS}$ activation, our reaction does not produce any nuisance waste products. The typical crude reaction mixture upon workup cleanly contains the desired alkyl chloride, although in most cases, a minor elimination product $(<10 \%)$ was detected by crude GC-MS or ${ }^{1} \mathrm{H}$ NMR analyses.

As shown in Table 3 entries 1-3, simple secondary alcohols in both acyclic and cyclic forms $\mathbf{8 a}-\mathbf{c}$ were readily converted to their corresponding secondary alkyl chlorides $\mathbf{9 a - c}$ in excellent yields. The use of enantiomerically pure alcohol 8a produced optically active $\mathbf{9 a}$ in high yield. The absolute configuration of this secondary chloride and its enantiomeric purity were determined by comparing its optical rotation to the literature value. ${ }^{23}$ These results confirmed that our chlorination reaction is stereospecific and proceeds via an inversion of 
stereo-chemistry. Our chlorination conditions proved to be mild, as $\beta$-hydroxy ester $\mathbf{8 d}$ readily afforded $\beta$-chloro ester $9 d$ in $82 \%$ yield without suffering from any substantial elimination. Olefins were also found to be a suitable functionality. Secondary alcohols $\mathbf{8 e , f}$, containing internal and terminal olefins, provided their corresponding chlorides $9 e, \mathbf{f}$ in good yields. Common protecting groups were also compatible under the reaction conditions. Entries 7-10 show that tert-butyldimethylsilyl ether, benzyl ether, $p$-methoxybenzyl ether, and $p$-toluoyl containing starting materials $\mathbf{8 g}-\mathbf{j}$ proceeded to chlorination without complications to give the secondary chlorides $\mathbf{9 g}-\mathbf{j}$ in excellent yields.

Our previous report also revealed the incompatibility of $a$-branched primary alcohols with our triphosgene-triethylamine chlorination conditions. Such substrates were readily transformed to the diethylcarbamate functionality. ${ }^{9}$ This problem was readily rectified with the new triphosgene-pyridine conditions. For example, as shown in Table 4, $\alpha$-phenyl and $a$-methyl phenethyl alcohols $\mathbf{1 0 a}, \mathbf{b}$ readily underwent chlorination to provide the primary alkyl chlorides 11a,b in $81 \%$ and $86 \%$ yields, respectively. $N$-Boc prolinol 10c also produced the corresponding chloride 11c in $65 \%$ yield.

As an extension to the scope of substrate study, we continued our exploration to demonstrate the feasibility of our method to simultaneously introduce two carbon-chlorine bonds via global chlorination of multiple hydroxy centers. Scheme 2 details our initial attempts at this strategy using starting materials such as 1,3-diol 12 and 1,6-diol 14. Treatment of these compounds by doubling the amount of triphosgene and pyridine cleanly afforded alkyl dichlorides 13 and $\mathbf{1 5}$ in 55\% and 73\% yields, respectively. The lower isolated yields were most likely attributed to the high volatility of the resulting alkyl chlorides. These results suggested that, in the presence of excess triphosgene and pyridine, global chloroformylation of the two hydroxy groups preceded the potentially competitive intramolecular sixmembered carbonate cyclization resulting from monochloroformylation. ${ }^{24,25}$ This hypothesis was clearly supported by the fact that exposure of 1,3-diol $\mathbf{1 2}$ to the typical 0.5 equiv of triphosgene and 2.0 equiv of pyridine resulted in a mixture of chlorination at the primary position, i.e. chloro alcohol 16, in 14\% yield and cyclic carbonate 17 in $66 \%$ yield. These observations were consistent with our previous report. ${ }^{9}$

The mechanism of this chlorination reaction is proposed as follows (Scheme 3). It is well precedented that activation of alcohol with a triphosgene-pyridine mixture leads to formation of the chloroformate functionality: viz., $18 .{ }^{16,19-22}$ It is reasonable to presume that excess pyridine then readily adds to the chloroformate to generate the putative $N$ acylpyridinium ion intermediate 19. ${ }^{26-28}$ This carbonyl activation increases the reactivity at the electrophilic secondary carbon center, which allows for $\mathrm{S}_{\mathrm{N}} 2$ nucleophilic substitution by chloride ions. This process releases $\mathrm{CO}_{2}$ and pyridine, while generating the alkyl chloride with an inversion of stereochemistry.

\section{CONCLUSION}

In summary, we have successfully addressed the previously unresolved issues concerning chlorination of aliphatic alcohols. We discovered that activation of unactivated aliphatic secondary alcohols with triphosgene and pyridine in dichloromethane at reflux cleanly 
produced the desired alkyl chlorides while eliminating the diethylcarbamoylation byproducts. These new reaction conditions are very useful and will strongly compliment the already existing chlorination methods. Our method is operationally simple, high yielding, stereospecific, mild, and well tolerated by a wide array of common functional and protecting groups. More importantly, our chlorination reaction does not produce any reactive or nuisance waste products, and the typical crude materials cleanly contain the target alkyl chloride. An extension of this work to other halogenation reactions and their applications toward stereoselective syntheses of chlorine-containing complex natural products are ongoing in our laboratories. Results from these efforts will be reported in due course.

\section{EXPERIMENTAL SECTION}

All materials, unless otherwise stated, were purchased from commercial sources and utilized without further purification. Anhydrous reactions were conducted in oven-dried glassware, which was then cooled under vacuum and purged with nitrogen gas. Anhydrous solvents (dichloromethane, toluene, acetonitrile, diethyl ether, and tetrahydrofuran) were filtered through activated $3 \AA$ molecular sieves under nitrogen in a solvent purification system. Reactions were monitored either by analytical thin-layer chromatography (TLC silica gel 60 $\mathrm{F}_{254}$, glass plates) and analyzed using $254 \mathrm{~nm}$ UV light and anisaldehyde-sulfuric acid or potassium permanganate stains or via gas chromatography-mass spectrometry (GC-MS). The column for the GC-MS system was 5\% phenyl methyl siloxane, measuring $30 \mathrm{~m}$ in length with an internal diameter of $250 \mu \mathrm{m}$ and film thickness of $0.25 \mu \mathrm{m}$. Low and high mass readings were set to 40 to $800 \mathrm{~m} / \mathrm{z}$, respectively. Oven, inlet, and detector temperatures were set to $250{ }^{\circ} \mathrm{C}$, and helium was used as the inert carrier gas. Column chromatography was completed using silica gel. Unless otherwise noted, all ${ }^{1} \mathrm{H}$ and ${ }^{13} \mathrm{CNMR}$ spectra were recorded in $\mathrm{CDCl}_{3}$ using a spectrometer operating at $400 \mathrm{MHz}$ for ${ }^{1} \mathrm{H}$ and $100 \mathrm{MHz}$ for ${ }^{13} \mathrm{C}$ or at $250 \mathrm{MHz}$ for ${ }^{1} \mathrm{H}$ and $62.5 \mathrm{MHz}$ for ${ }^{13} \mathrm{C}$. Chemical shifts $(\delta)$ are reported in ppm relative to residual $\mathrm{CHCl}_{3}$ as an internal reference $\left({ }^{1} \mathrm{H}, 7.26 \mathrm{ppm} ;{ }^{13} \mathrm{C}, 77.00 \mathrm{ppm}\right)$. Coupling constants $(\mathbf{J})$ are reported in hertz $(\mathrm{Hz})$. Peak multiplicity is indicated as follows: $\mathrm{s}$ (singlet), d (doublet), t (triplet), q (quartet), p (pentet), x (septet), h (heptet), b (broad), and $\mathrm{m}$ (multiplet). FT-IR spectra were recorded using thin films, and absorption frequencies are reported in reciprocal centimeters $\left(\mathrm{cm}^{-1}\right)$. High-resolution mass spectrometry (HRMS) analyses were performed using electron spray ionization-time of flight (ESI-TOF) methods.

\section{General Chlorination Procedure}

Unless otherwise noted, the alcohol $(2.0 \mathrm{mmol})$ was placed in an oven-dried round-bottomed flask and dissolved in anhydrous dichloromethane $(15 \mathrm{~mL})$. The solution was then cooled to $0{ }^{\circ} \mathrm{C}$. Pyridine $(0.32 \mathrm{~mL}, 4.0 \mathrm{mmol})$ was then added via syringe, followed by triphosgene ( $297 \mathrm{mg}, 1.0 \mathrm{mmol}$ ) in one portion. The solution was stirred for $5 \mathrm{~min}$ and then warmed to gentle reflux overnight. The reaction mixture was then poured into a separatory funnel containing $1 \mathrm{M} \mathrm{HCl}$ aqueous solution $(30 \mathrm{~mL})$, and the biphasic mixture was shaken vigorously. Upon separation of layers, the aqueous layer was re-extracted with dichloromethane $(2 \times 30 \mathrm{~mL})$. Organic extracts were collected, dried over $\mathrm{MgSO}_{4}$, filtered, and concentrated under vacuum. The resulting crude material was purified using flash 
column chromatography with silica gel as the stationary phase and a hexanes/ethyl acetate, pentane/diethyl ether, or pentane/dichloromethane mixture as the mobile phase.

(+)-(S)-(2-Chloropropyl)benzene (9a)-Alcohol 8a (272 mg, $2.00 \mathrm{mmol})$ was utilized along with pyridine $(0.32 \mathrm{~mL}, 4.00 \mathrm{mmol})$ and triphosgene $(297 \mathrm{mg}, 1.00 \mathrm{mmol})$ to produce 9a in $89 \%$ yield as a colorless oil ( $273 \mathrm{mg}, 1.77 \mathrm{mmol})$. The purified product was eluted with $100 \%$ hexanes. ${ }^{1} \mathrm{H}$ NMR $\left(250 \mathrm{MHz}, \mathrm{CDCl}_{3}\right): \delta(\mathrm{ppm}) 7.37-7.21(5 \mathrm{H}, \mathrm{m}), 4.24(1 \mathrm{H}, \mathrm{x}$, $J=6.8 \mathrm{~Hz}), 3.11(1 \mathrm{H}, \mathrm{dd}, J=13.8,7.0 \mathrm{~Hz}), 2.98(1 \mathrm{H}, \mathrm{dd}, J=13.9,7.0 \mathrm{~Hz}), 1.53(3 \mathrm{H}, \mathrm{d}, J=$ $6.5 \mathrm{~Hz}) .{ }^{13} \mathrm{C} \mathrm{NMR}\left(62.5 \mathrm{MHz}, \mathrm{CDCl}_{3}\right): \delta(\mathrm{ppm}) 137.9,129.2,128.3,126.7,58.4,46.6,24.6$. GC-MS: $\mathrm{M}^{+} 154.6$ calculated for $\mathrm{C}_{9} \mathrm{H}_{11} \mathrm{Cl}$, experimental 154.0. $[a]_{25} \mathrm{D}=+23.12^{\circ}(c=2.2$ in $\mathrm{CHCl})$. Compound 9a is known: ${ }^{23}$ literature $[a]_{25}{ }^{\mathrm{D}}=+23.19^{\circ}\left(c=5\right.$ in $\left.\mathrm{CHCl}_{3}\right)$ for enantiomerically pure $(S)$-(2-chloropropyl)benzene.

(2-Chloropropane-1,3-diyl)dibenzene (9b)_Alcohol $8 \mathbf{b}$ (420 mg, $2.00 \mathrm{mmol}$ ) was utilized along with pyridine $(0.32 \mathrm{~mL}, 4.00 \mathrm{mmol})$ and triphosgene $(297 \mathrm{mg}, 1.00 \mathrm{mmol})$ to produce $9 \mathrm{~b}$ in $87 \%$ yield as a colorless oil $(397 \mathrm{mg}, 1.73 \mathrm{mmol})$. The purified product was eluted with $100 / 0 \rightarrow 90 / 10$ hexanes/EtOAc. ${ }^{1} \mathrm{H}$ NMR $\left(250 \mathrm{MHz}, \mathrm{CDCl}_{3}\right): \delta(\mathrm{ppm}) 7.38-$ $7.23(10 \mathrm{H}, \mathrm{m}), 4.34(1 \mathrm{H}, \mathrm{dddd}, J=8.0,8.0,5.6,5.2 \mathrm{~Hz}), 3.16(2 \mathrm{H}, \mathrm{dd}, J=14.1,5.6 \mathrm{~Hz})$, $3.04(2 \mathrm{H}, \mathrm{dd}, J=14.2,8.0 \mathrm{~Hz}) .{ }^{13} \mathrm{C} \mathrm{NMR}\left(62.5 \mathrm{MHz}, \mathrm{CDCl}_{3}\right): \delta(\mathrm{ppm}) 137.8,129.3,128.3$, 126.7, 63.9, 44.2. IR $\left(\mathrm{cm}^{-1}\right): v 3088,3064,3029,1603,1498,1455,911,741,700,670$. GC-MS: $(\mathrm{M}-\mathrm{H})^{+} 229.7$ calculated for $\mathrm{C}_{15} \mathrm{H}_{14} \mathrm{Cl}$, experimental 229.9. Compound $9 \mathbf{b}$ is known. ${ }^{29}$

N-Boc-4-chloropiperidine (9c) -Alcohol 8c (403 mg, $2.00 \mathrm{mmol})$ was utilized along with pyridine $(0.32 \mathrm{~mL}, 4.00 \mathrm{mmol})$ and triphosgene $(297 \mathrm{mg}, 1.00 \mathrm{mmol})$ to produce $9 \mathrm{c}$ in $88 \%$ yield as a pale yellow oil $(387 \mathrm{mg}, 1.76 \mathrm{mmol})$. The purified product was eluted with 90/10 $\rightarrow 80 / 20$ pentane/diethyl ether. ${ }^{1} \mathrm{H}$ NMR $\left(400 \mathrm{MHz}, \mathrm{CDCl}_{3}\right): \delta(\mathrm{ppm}) 4.20(1 \mathrm{H}, \mathrm{m})$, 3.73-3.68 (2H, m), 3.32-3.26 (2H, m) 2.06-1.99 (2H, m), 1.86-1.76 (2H, m), $1.46(9 \mathrm{H}$, s). ${ }^{13} \mathrm{C} \mathrm{NMR}\left(100 \mathrm{MHz}, \mathrm{CDCl}_{3}\right): \delta$ (ppm) $154.5,79.6,56.8,41.3$ (b), 34.9, 28.3. IR $\left(\mathrm{cm}^{-1}\right)$ : $v 2977,2870,2839,1692,1478,1419,1366,1264,1218,1165,1110,1001,895,767,719$. HRMS-ESI: $(\mathrm{M}+\mathrm{Na})^{+} 242.0918$ calculated for $\mathrm{C}_{10} \mathrm{H}_{18} \mathrm{ClNNaO}_{2}$, experimental 242.0918 .

Ethyl (士)-3-Chloro-4-phenylbutyrate (9d)—Alcohol 8d ${ }^{30}$ (417 mg, $2.00 \mathrm{mmol}$ ) was utilized along with pyridine $(0.32 \mathrm{~mL}, 4.00 \mathrm{mmol})$ and triphosgene $(297 \mathrm{mg}, 1.00 \mathrm{mmol})$ to produce $9 \mathrm{~d}$ in $82 \%$ yield as a pale yellow oil $(372 \mathrm{mg}, 1.64 \mathrm{mmol})$. The purified product was eluted with $100 \%$ pentane. ${ }^{1} \mathrm{H}$ NMR $\left(400 \mathrm{MHz}, \mathrm{CDCl}_{3}\right): \delta(\mathrm{ppm}) 7.34-7.22(5 \mathrm{H}, \mathrm{m})$, $4.51(1 \mathrm{H}, \mathrm{dt}, J=13.4,7.3 \mathrm{~Hz}), 4.16 \mathrm{ppm}(2 \mathrm{H}, \mathrm{q}, J=6.8 \mathrm{~Hz}), 3.09(2 \mathrm{H}, \mathrm{dd}, J=6.7,4.6 \mathrm{~Hz})$, 2.75-2.71 (2H, m), $1.27(3 \mathrm{H}, \mathrm{t}, J=7.1 \mathrm{~Hz}) .{ }^{13} \mathrm{C} \mathrm{NMR}\left(100 \mathrm{MHz}, \mathrm{CDCl}_{3}\right): \delta(\mathrm{ppm}) 170.1$, 137.0, 129.5, 128.6, 127.1, 60.9, 57.7, 44.3, 42.6, 14.2. IR ( $\left.\mathrm{cm}^{-1}\right): v 3065,3030,2983$, 2905, 1737, 1654, 1304, 1150, 1096, 910, 747, 650. HRMS-ESI: $(\mathrm{M}+\mathrm{H})^{+} 227.0833$ calculated for $\mathrm{C}_{12} \mathrm{H}_{16} \mathrm{ClO}_{2}$, experimental 227.0835.

( \pm )-4-Chloro-5-phenylpent-1-ene (9e)-Alcohol 8e ${ }^{31}$ (324 mg, 2.00 mmol) was utilized along with pyridine $(0.32 \mathrm{~mL}, 4.00 \mathrm{mmol})$ and triphosgene $(297 \mathrm{mg}, 1.00 \mathrm{mmol})$ to produce $9 \mathrm{e}$ in $94 \%$ yield as a colorless oil $(340 \mathrm{mg}, 1.88 \mathrm{mmol})$. The purified product was 
eluted with $100 \%$ pentane. ${ }^{1} \mathrm{H}$ NMR (400, $\left.\mathrm{MHz}, \mathrm{CDCl}_{3}\right): \delta(\mathrm{ppm}) 7.36-7.24(5 \mathrm{H}, \mathrm{m}), 5.93$ $(1 \mathrm{H}, \mathrm{m}), 5.20-5.15(2 \mathrm{H}, \mathrm{m}), 4.17(1 \mathrm{H}, \mathrm{m}), 3.10(1 \mathrm{H}, \mathrm{dd}, J=14.1,6.2 \mathrm{~Hz}), 3.04(1 \mathrm{H}, \mathrm{dd}, J=$ 14.1, $6.4 \mathrm{~Hz}), 2.59(1 \mathrm{H}, \mathrm{m}), 2.48(1 \mathrm{H}, \mathrm{m}) .{ }^{13} \mathrm{C} \mathrm{NMR}\left(100 \mathrm{MHz}, \mathrm{CDCl}_{3}\right): \delta(\mathrm{ppm}) 137.8$, 134.0, 129.4, 128.5, 126.8, 118.3, 62.5, 44.2, 41.8. IR ( $\left.\mathrm{cm}^{-1}\right): v 3080,3030,2981,2952$, 1643, 1604, 1543, 1433, 1284, 1031, 993, 920, 700, 618. GC-MS: $\mathrm{M}^{+} 180.1$ calculated for $\mathrm{C}_{11} \mathrm{H}_{13} \mathrm{Cl}$, experimental 180.0. Compound 9e is known. ${ }^{6}$

( \pm )-(E)-4-Chloro-1-phenylhepta-1,6-diene (9f)—Alcohol $8 \mathbf{f}$ (377 mg, $2.00 \mathrm{mmol}$ ) was utilized along with pyridine $(0.32 \mathrm{~mL}, 4.00 \mathrm{mmol})$ and triphosgene $(297 \mathrm{mg}, 1.00 \mathrm{mmol})$ to produce $9 \mathrm{f}$ in $82 \%$ yield as a yellow oil ( $339 \mathrm{mg}, 1.64 \mathrm{mmol})$. The purified product was eluted with $100 \%$ pentane. ${ }^{1} \mathrm{H} \mathrm{NMR}\left(400 \mathrm{MHz}, \mathrm{CDCl}_{3}\right): \delta(\mathrm{ppm}) 7.38-7.17(5 \mathrm{H}, \mathrm{m}), 6.48$ $(1 \mathrm{H}, \mathrm{d}, J=15.8 \mathrm{~Hz}), 6.25(1 \mathrm{H}, \mathrm{m}), 5.94-5.83(1 \mathrm{H}, \mathrm{m}), 5.18-5.10(2 \mathrm{H}, \mathrm{m}), 4.03(1 \mathrm{H}, \mathrm{m})$, 2.72-2.61 (2H, m), 2.59-2.50 (2H, m). $\left.{ }^{13} \mathrm{C} \mathrm{NMR} \mathrm{(100} \mathrm{MHz,} \mathrm{CDCl}_{3}\right): \delta(\mathrm{ppm}) 137.1,134.0$, 133.2, 128.6, 127.4, 126.2, 125.5, 118.2, 61.5, 42.0, 41.2. IR ( $\left.\mathrm{cm}^{-1}\right): v 3081,3061,3028$, 2980, 2946, 1644, 1495, 1449, 1289, 967, 918, 744, 694. HRMS-ESI: $(\mathrm{M}+\mathrm{H})^{+} 207.0935$ calculated for $\mathrm{C}_{13} \mathrm{H}_{16} \mathrm{Cl}$, experimental 207.0936.

( \pm )-4-((tert-Butyldimethylsilyl)oxy)-2-chloro-1-phenylbutane (9g)-Alcohol 8g $(560 \mathrm{mg}, 2.00 \mathrm{mmol})$ was utilized along with pyridine $(0.32 \mathrm{~mL}, 4.00 \mathrm{mmol})$ and triphosgene (297 mg, $1.00 \mathrm{mmol}$ ) to produce $9 \mathrm{~g}$ in $90 \%$ yield as a colorless oil $(536 \mathrm{mg}$, $1.80 \mathrm{mmol})$. The purified product was eluted with $100 / 0 \rightarrow 90 / 10$ hexanes/EtOAc. ${ }^{1} \mathrm{H}$ NMR $\left(400 \mathrm{MHz}, \mathrm{CDCl}_{3}\right): \delta(\mathrm{ppm}) 7.36-7.22(5 \mathrm{H}, \mathrm{m}), 4.33(1 \mathrm{H}, \mathrm{ddt}, J=10.1,9.8,3.4 \mathrm{~Hz}), 3.85-$ $3.73(2 \mathrm{H}, \mathrm{m}), 3.08(2 \mathrm{H}, \mathrm{d}, J=6.8 \mathrm{~Hz}), 2.03(1 \mathrm{H}, \mathrm{m}), 1.81(1 \mathrm{H}, \mathrm{ddt}, J=14.3,9.8,4.4 \mathrm{~Hz})$, $0.89(9 \mathrm{H}, \mathrm{s}), 0.05(6 \mathrm{H}, \mathrm{d}, J=7.1 \mathrm{~Hz}) .{ }^{13} \mathrm{C}$ NMR $\left(100 \mathrm{MHz}, \mathrm{CDCl}_{3}\right): \delta(\mathrm{ppm}) 137.9,129.4$, 128.4, 126.7, 60.3, 59.8, 45.1, 40.6, 25.9, 18.3, -5.4. IR $\left(\mathrm{cm}^{-1}\right): v 3030,2954,2930,2857$, 1472, 1256, 1110, 910, 837, 778. HRMS-ESI: $(\mathrm{M}+\mathrm{H})^{+} 299.1592$ calculated for $\mathrm{C}_{16} \mathrm{H}_{28} \mathrm{ClOSi}$, experimental 299.1599.

(士)-4-Benzyloxy-2-chloro-1-phenylbutane (9h)—Alcohol $8 \mathrm{~h}$ (256 mg, $2.00 \mathrm{mmol}$ ) was utilized along with pyridine $(0.32 \mathrm{~mL}, 4.00 \mathrm{mmol})$ and triphosgene $(297 \mathrm{mg}, 1.00$ $\mathrm{mmol}$ ) to produce $\mathbf{9 h}$ in $84 \%$ yield as a colorless oil $(462 \mathrm{mg}, 1.69 \mathrm{mmol})$. The purified product was eluted with 98/2 hexanes/EtOAc. ${ }^{1} \mathrm{H} \mathrm{NMR}\left(400 \mathrm{MHz}, \mathrm{CDCl}_{3}\right): \delta(\mathrm{ppm}) 7.34$ $7.21(10 \mathrm{H}, \mathrm{m}), 4.54-4.45(2 \mathrm{H}, \mathrm{m}), 3.88(1 \mathrm{H}, \mathrm{m}), 3.69-3.60(2 \mathrm{H}, \mathrm{m}), 2.97(1 \mathrm{H}, \mathrm{dd}, J=13.5$, $6.0 \mathrm{~Hz}), 2.80(1 \mathrm{H}, \mathrm{dd}, J=13.6,6.4 \mathrm{~Hz}), 1.96-1.90(2 \mathrm{H}, \mathrm{m}) .{ }^{13} \mathrm{C}$ NMR $\left(100, \mathrm{CDCl}_{3}\right): \delta$ (ppm) $138.3,138.1,129.5,128.4,128.0,127.8,126.4,77.1,72.1,41.7,40.6,37.4$. IR $\left(\mathrm{cm}^{-1}\right): v 3063,3023,2925,2866,1496,1456,1350,1289,1073,1029,910,737,699,651$. HRMS-ESI: $(\mathrm{M}+\mathrm{Na})^{+} 297.1017$ calculated for $\mathrm{C}_{17} \mathrm{H}_{19} \mathrm{ClNaO}$, experimental 297.1019.

( \pm )-2-Chloro-4-((4-methoxybenzyl)oxy)-1-phenylbutane (9i)-Alcohol 8i (572 mg, $2.00 \mathrm{mmol})$ was utilized along with pyridine $(0.32 \mathrm{~mL}, 4.00 \mathrm{mmol})$, and triphosgene (297 $\mathrm{mg}, 1.00 \mathrm{mmol}$ ) to produce $9 \mathbf{i}$ in $82 \%$ yield as a colorless oil (498 $\mathrm{mg}, 1.64 \mathrm{mmol})$. The purified product was eluted with 90/10 hexanes/EtOAc. ${ }^{1} \mathrm{H}$ NMR $\left(400 \mathrm{MHz}, \mathrm{CDCl}_{3}\right): \delta$ (ppm) 7.33-7.19 (7H, m), $6.87(2 \mathrm{H}, \mathrm{d}, J=8.6 \mathrm{~Hz}), 4.42(2 \mathrm{H}, \mathrm{q}, J=10.9 \mathrm{~Hz}), 3.86(1 \mathrm{H}, \mathrm{m})$, $3.81(3 \mathrm{H}, \mathrm{s}), 3.67-3.57(2 \mathrm{H}, \mathrm{m}), 2.95(1 \mathrm{H}, \mathrm{dd}, J=13.6,6.1 \mathrm{~Hz}), 2.78(1 \mathrm{H}, \mathrm{dd}, J=13.7,6.4$ $\mathrm{Hz}), 1.96-1.86(2 \mathrm{H}, \mathrm{m}) .{ }^{13} \mathrm{C} \mathrm{NMR}\left(100 \mathrm{MHz}, \mathrm{CDCl}_{3}\right): \delta(\mathrm{ppm}) 159.3,138.2,130.4,129.5$, 
128.4, 126.4, 113.9, 76.8, 71.8, 55.3, 41.7, 40.7, 37.4. IR ( $\left.\mathrm{cm}^{-1}\right): v 3063,3030,2936,2870$, 1612, 1513, 1248, 1076, 1034, 822, 740, 701. HRMS-ESI: $(\mathrm{M}+\mathrm{Na})^{+} 327.1122$ calculated for $\mathrm{C}_{18} \mathrm{H}_{21} \mathrm{ClNaO}_{2}$, experimental 327.1133.

( \pm )-3-Chloro-4-phenylbutyl 4-Methylbenzoate (9j)—Alcohol 8j (568 mg, $2.00 \mathrm{mmol}$ ) was utilized along with pyridine $(0.32 \mathrm{~mL}, 4.00 \mathrm{mmol})$ and triphosgene $(297 \mathrm{mg}, 1.00$ $\mathrm{mmol}$ ) to produce $\mathbf{9 j}$ in $85 \%$ yield as a colorless oil $(514 \mathrm{mg}, 1.70 \mathrm{mmol})$. The purified product was eluted with 90/10 hexanes/EtOAc. ${ }^{1} \mathrm{H}$ NMR (400 MHz, $\left.\mathrm{CDCl}_{3}\right): \delta(\mathrm{ppm}) 7.91$ $(2 \mathrm{H}, \mathrm{d}, J=8.2 \mathrm{~Hz}), 7.35-7.24(5 \mathrm{H}, \mathrm{m}), 4.55(1 \mathrm{H}, \mathrm{ddd}, J=11.2,6.2,4.7 \mathrm{~Hz}), 4.47(1 \mathrm{H}, \mathrm{m})$, $4.32(1 \mathrm{H}, \mathrm{m}), 3.13(2 \mathrm{H}, \mathrm{d}, J=6.8 \mathrm{~Hz}), 2.42(3 \mathrm{H}, \mathrm{s}), 2.31(1 \mathrm{H}, \mathrm{m}), 2.09(1 \mathrm{H}, \mathrm{m}) .{ }^{13} \mathrm{C}$ NMR $\left(100 \mathrm{MHz}, \mathrm{CDCl}_{3}\right): \delta(\mathrm{ppm}) 166.5,143.8,137.4,129.6,129.4,129.1,128.5,127.4,127.0$, 61.8, 59.9, 45.0, 36.7, 21.7. IR ( $\left.\mathrm{cm}^{-1}\right): v 3087,3062,2964,2861,1715,1270,1177,1109$, 1021, 752, 700. HRMS-ESI: $(\mathrm{M}+\mathrm{H})^{+} 303.1152$ calculated for $\mathrm{C}_{18} \mathrm{H}_{20} \mathrm{ClO}_{2}$, experimental 303.1145 .

2-Chloro-1,1-diphenylethane (11a)_Alcohol 10a (397 mg, $2.00 \mathrm{mmol}$ ) was utilized along with pyridine $(0.32 \mathrm{~mL}, 4.00 \mathrm{mmol})$ and triphosgene $(297 \mathrm{mg}, 1.00 \mathrm{mmol})$ to produce 11a in $81 \%$ yield as a light yellow oil $(350 \mathrm{mg}, 1.62 \mathrm{mmol})$. The purified product was eluted with $100 \%$ hexanes. ${ }^{1} \mathrm{H}$ NMR $\left(250 \mathrm{MHz}, \mathrm{CDCl}_{3}\right): \delta(\mathrm{ppm}) 7.35-7.21(10 \mathrm{H}, \mathrm{m}), 4.34(1 \mathrm{H}, \mathrm{t}$, $J=7.8 \mathrm{~Hz}), 4.07(2 \mathrm{H}, \mathrm{d}, J=7.8 \mathrm{~Hz}) .{ }^{13} \mathrm{C} \mathrm{NMR}\left(62.5 \mathrm{MHz}, \mathrm{CDCl}_{3}\right): \delta(\mathrm{ppm}) 141.2,128.5$, 127.9, 126.9, 53.5, 47.1. IR $\left(\mathrm{cm}^{-1}\right): v 3062,3029,1494,1452,910,737,699$. GC-MS: $\mathrm{M}^{+}$ 216.1 calculated for $\mathrm{C}_{14} \mathrm{H}_{13} \mathrm{Cl}$, experimental 216.0. Compound 11a is known. ${ }^{32}$

(士)-1-Chloro-2-phenylpropane (11b)—Alcohol 10b $(0.28 \mathrm{~mL}, 2.00 \mathrm{mmol})$ was utilized along with pyridine $(0.32 \mathrm{~mL}, 4.00 \mathrm{mmol})$ and triphosgene $(297 \mathrm{mg}, 1.00 \mathrm{mmol})$ to produce $11 \mathrm{~b}$ in $86 \%$ yield as a colorless oil $(266 \mathrm{mg}, 1.73 \mathrm{mmol})$. The purified product was eluted with $100 \%$ hexanes. ${ }^{1} \mathrm{H}$ NMR $\left(250 \mathrm{MHz}, \mathrm{CDCl}_{3}\right): \delta(\mathrm{ppm}) 7.39-7.19(5 \mathrm{H}, \mathrm{m}), 3.72(1 \mathrm{H}$, dd, $J=10.7,6.2 \mathrm{~Hz}), 3.61(1 \mathrm{H}, \mathrm{dd}, J=10.7,7.8 \mathrm{~Hz}), 3.12(1 \mathrm{H}, \mathrm{x}, J=7.0 \mathrm{~Hz}), 1.42(3 \mathrm{H}, \mathrm{d}, J=$ $7.0 \mathrm{~Hz}) .{ }^{13} \mathrm{C}$ NMR $\left(62.5 \mathrm{MHz}, \mathrm{CDCl}_{3}\right): \delta(\mathrm{ppm}) 143.2,128.5,127.1,126.8,50.7,42.2,18.9$. IR $\left(\mathrm{cm}^{-1}\right): v 3030,2970,2875,1494,1454,1015,910,762,720,699$. GC-MS: (M) ${ }^{+} 154.1$ calculated for $\mathrm{C}_{9} \mathrm{H}_{11} \mathrm{Cl}$, experimental 154.0. Compound $\mathbf{1 1 b}$ is available commercially.

(S)-N-Boc-2-(chloromethyl)pyrrolidine (11c)_Alcohol 10c (345 mg, $2.00 \mathrm{mmol}$ ) was utilized along with pyridine $(0.32 \mathrm{~mL}, 4.00 \mathrm{mmol})$ and triphosgene $(297 \mathrm{mg}, 1.00 \mathrm{mmol})$ to produce $11 \mathrm{c}$ in $65 \%$ yield as a colorless oil $(244 \mathrm{mg}, 1.11 \mathrm{mmol})$. The purified product was eluted with 90/10 hexanes/EtOAc. ${ }^{1} \mathrm{H}$ NMR $\left(400 \mathrm{MHz}, \mathrm{CDCl}_{3}\right): \delta(\mathrm{ppm}) 4.04(0.5 \mathrm{H}, \mathrm{b})$, $3.95(0.5 \mathrm{H}, \mathrm{b}), 3.75(0.5 \mathrm{H}, \mathrm{b}, \mathrm{d}, J=9.9 \mathrm{~Hz}), 3.66(0.5 \mathrm{H}, \mathrm{b}, \mathrm{d}, J=9.9 \mathrm{~Hz}), 3.54(0.5 \mathrm{H}, \mathrm{t}, J=$ $9.0 \mathrm{~Hz}), 3.46-3.33(2.5 \mathrm{H}, \mathrm{m}), 2.00(2 \mathrm{H}, \mathrm{t}, J=6.4 \mathrm{~Hz}), 1.88-1.78(2 \mathrm{H}, \mathrm{m}), 1.46(9 \mathrm{H}, \mathrm{s}) .{ }^{13} \mathrm{C}$ NMR (100, $\left.\mathrm{MHz}, \mathrm{CDCl}_{3}\right): \delta(\mathrm{ppm}) 154.5,154.2,79.8,79.5,58.1,58.0,47.3,46.8,45.5$, 45.3, 29.2, 28.4, 23.6, 22.8. IR ( $\left.\mathrm{cm}^{-1}\right): v 2977,2879,1695,1392,1171,1118,910,733$. $[a]_{25} \mathrm{D}=-7.29^{\circ}(c=1.6$ in $\mathrm{CHCl})$. HRMS-ESI: $(\mathrm{M}+\mathrm{Na})^{+} 242.0924$ calculated for $\mathrm{C}_{10} \mathrm{H}_{18} \mathrm{ClNNaO}_{2}$, experimental 242.0917 .

( \pm )-(2,4-Dichlorobutyl)benzene (13)—Alcohol $12{ }^{33}$ (332 mg, $\left.2.00 \mathrm{mmol}\right)$ was utilized along with pyridine $(0.65 \mathrm{~mL}, 8.00 \mathrm{mmol})$ and triphosgene $(593 \mathrm{mg}, 2.00 \mathrm{mmol})$ in $30 \mathrm{~mL}$ 
of $\mathrm{CH}_{2} \mathrm{Cl}_{2}$ to produce 13 in $55 \%$ yield as a colorless oil ( $\left.223 \mathrm{mg}, 1.10 \mathrm{mmol}\right)$. The purified product was eluted with 100/0 $\rightarrow$ 90/10 hexanes/EtOAc. ${ }^{1} \mathrm{H}$ NMR (400 MHz, $\mathrm{CDCl}_{3}$ ): $\delta$ (ppm) 7.36-7.22 (5H, m), $4.35(1 \mathrm{H}, \mathrm{m}), 3.78-3.69(2 \mathrm{H}, \mathrm{m}), 3.13(1 \mathrm{H}, \mathrm{dd}, J=14.1,7.3 \mathrm{~Hz})$, $3.06(1 \mathrm{H}, \mathrm{dd}, J=14.0,6.6 \mathrm{~Hz}), 2.20(1 \mathrm{H}, \mathrm{m}), 2.08(1 \mathrm{H}, \mathrm{m}) .{ }^{13} \mathrm{C} \mathrm{NMR}\left(100 \mathrm{MHz}, \mathrm{CDCl}_{3}\right): \delta$ (ppm) 137.1, 129.4, 128.5, 127.0, 60.0, 44.8, 41.7, 40.1. IR ( $\left.\mathrm{cm}^{-1}\right): v 3031,2965,1496$, $1454,1318,1285,910,735,701$. GC-MS: $\mathrm{M}^{+} 202.0$ calculated for $\mathrm{C}_{10} \mathrm{H}_{12} \mathrm{Cl}_{2}$, experimental 202.0. HRMS-ESI: $(\mathrm{M}-\mathrm{H}-2 \mathrm{Cl})^{+} 131.0855$ calculated for $\mathrm{C}_{10} \mathrm{H}_{11}$, experimental 131.0857.

(士)-4,8-Dichlorooct-1-ene (15)—Alcohol $14^{34}$ (288 mg, $\left.2.00 \mathrm{mmol}\right)$ was utilized along with pyridine $(0.64 \mathrm{~mL}, 8.00 \mathrm{mmol})$ and triphosgene $(593 \mathrm{mg}, 2.00 \mathrm{mmol})$ in $30 \mathrm{~mL}$ of $\mathrm{CH}_{2} \mathrm{Cl}_{2}$ to produce $15 \mathrm{in} 73 \%$ yield as a colorless oil $(264 \mathrm{mg}, 1.47 \mathrm{mmol})$. The purified product was eluted with 100/0 $\rightarrow$ 90/10 hexanes/EtOAc. ${ }^{1} \mathrm{H}$ NMR $\left(400 \mathrm{MHz}, \mathrm{CDCl}_{3}\right): \delta$ (ppm) $5.85(1 \mathrm{H}, \mathrm{m}), 5.16-5.12(2 \mathrm{H}, \mathrm{m}), 3.93(1 \mathrm{H}, \mathrm{m}), 3.55(2 \mathrm{H}, \mathrm{t}, J=6.4 \mathrm{~Hz}), 2.53-2.49$ $(2 \mathrm{H}, \mathrm{m}), 1.87-1.66(6 \mathrm{H}, \mathrm{m}) .{ }^{13} \mathrm{C} \mathrm{NMR}\left(100 \mathrm{MHz}, \mathrm{CDCl}_{3}\right): \delta(\mathrm{ppm}) 134.0,118.1,62.2$, 44.7, 42.7, 37.0, 32.1, 23.8. IR $\left(\mathrm{cm}^{-1}\right): v 3081,2950,2868,1643,995,911,735,650$. GCMS: $(\mathrm{M})^{+} 180.0$ calculated for $\mathrm{C}_{8} \mathrm{H}_{14} \mathrm{Cl}_{2}$, experimental 179.9. HRMS-ESI: $(\mathrm{M}-2 \mathrm{H}-2 \mathrm{Cl}+$ $\mathrm{Na})^{+} 131.0831$ calculated for $\mathrm{C}_{8} \mathrm{H}_{12} \mathrm{Na}$, experimental 131.0838 .

(士)-4-Chloro-1-phenylbutan-2-ol (16) and ( \pm )-4-Benzyl-1,3-dioxan-2-one (17)Alcohol $12{ }^{33}$ (332 mg, $\left.2.00 \mathrm{mmol}\right)$ was utilized along with pyridine $(0.32 \mathrm{~mL}, 4.00 \mathrm{mmol})$ and triphosgene ( $297 \mathrm{mg}, 1.00 \mathrm{mmol}$ ) to produce $17 \mathrm{in} 66 \%$ yield as a colorless oil (254 mg, $1.32 \mathrm{mmol}$ ) and 16 in $14 \%$ yield as a colorless oil $(50 \mathrm{mg}, 0.27 \mathrm{mmol})$. The purified products were eluted with $90 / 10 \rightarrow 80 / 20 \rightarrow 70 / 30$ hexanes/EtOAc. Data for the less polar product (16) are as follows. ${ }^{1} \mathrm{H}$ NMR $\left(400 \mathrm{MHz}, \mathrm{CDCl}_{3}\right): \delta(\mathrm{ppm}) 7.38-7.22(5 \mathrm{H}, \mathrm{m}), 4.08$ $(1 \mathrm{H}, \mathrm{ddd}, J=15.8,7.9,4.7 \mathrm{~Hz}), 3.80-3.65(2 \mathrm{H}, \mathrm{m}), 2.86(1 \mathrm{H}, \mathrm{dd}, J=13.5,4.2 \mathrm{~Hz}), 2.71$ $(1 \mathrm{H}, \mathrm{dd}, J=13.5,8.6 \mathrm{~Hz}), 2.01-1.93(2 \mathrm{H}, \mathrm{m}), 1.66(1 \mathrm{H}, \mathrm{d}, J=3.0 \mathrm{~Hz}) .{ }^{13} \mathrm{C}$ NMR $(100$ $\left.\mathrm{MHz}, \mathrm{CDCl}_{3}\right): \delta(\mathrm{ppm}) 137.7,129.3,129.6,126.6,69.4,43.9,41.7,39.1 . \mathrm{IR}\left(\mathrm{cm}^{-1}\right): v 3425$, 3378, 3063, 3029, 2943, 2920, 1946, 1454, 1081, 742, 701. HRMS-ESI: $(\mathrm{M}+\mathrm{Na})^{+}$ 207.0553 calculated for $\mathrm{C}_{10} \mathrm{H}_{13} \mathrm{ClNaO}$, experimental 207.0543. Data for the more polar product (17) are as follows. ${ }^{1} \mathrm{H} \mathrm{NMR}\left(400 \mathrm{MHz}, \mathrm{CDCl}_{3}\right): \delta(\mathrm{ppm}) 7.37-7.22(5 \mathrm{H}, \mathrm{m}), 4.67$ $(1 \mathrm{H}, \mathrm{m}), 4.45-4.27(2 \mathrm{H}, \mathrm{m}), 3.15(1 \mathrm{H}, \mathrm{dd}, J=13.8,5.7 \mathrm{~Hz}), 2.92(1 \mathrm{H}, \mathrm{dd}, J=13.8,7.3 \mathrm{~Hz})$, 2.03-1.83 (2H, m). $\left.{ }^{13} \mathrm{C} \mathrm{NMR} \mathrm{(100} \mathrm{MHz,} \mathrm{CDCl}_{3}\right): \delta(\mathrm{ppm}) 148.6,135.0,129.4,128.7$, 127.1, 79.5, 66.8, 41.3, 26.2. IR ( $\left.\mathrm{cm}^{-1}\right): v 3352,3028,2973,2932,2875,1753,1409,1250$, 1188, 1124, 911, 738, 703. HRMS-ESI: $(\mathrm{M}-\mathrm{CO}+3 \mathrm{H})^{+} 167.1072$ calculated for $\mathrm{C}_{10} \mathrm{H}_{15} \mathrm{O}_{2}$, experimental 167.1055.

\section{Preparation of Secondary Alcohols $8 f-j$} (士)-(E)-1-Phenylhepta-1,6-dien-4-ol (8f)-

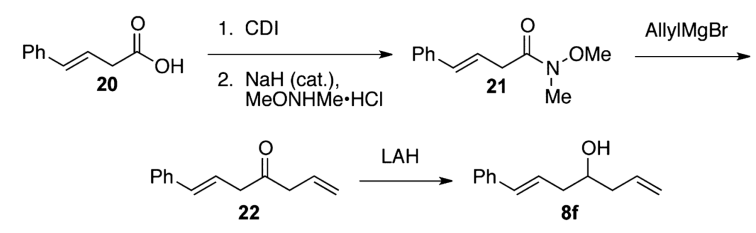


trans-Styrylacetic acid (20; $3.30 \mathrm{~g}, 20.35 \mathrm{mmol})$ was dissolved in THF $(50 \mathrm{~mL})$, and carbonyldiimidazole $(4.30 \mathrm{~g}, 26.45 \mathrm{mmol})$ was then added in one portion. The reaction mixture was then stirred overnight. The crude reaction mixture was concentrated in vacuo, diluted with $\mathrm{Et}_{2} \mathrm{O}(50 \mathrm{~mL})$, and then washed with saturated brine solution $(3 \times 50 \mathrm{~mL})$. Collected aqueous layers were extracted with $\mathrm{Et}_{2} \mathrm{O}(3 \times 50 \mathrm{~mL})$ and combined with the organic layer. The organic fractions were then dried over $\mathrm{MgSO}_{4}$, concentrated under vacuum, and taken to the next step without further purification. This crude material was then dissolved in THF (50 mL), and MeONHMe $\mathrm{HCl}(1.85 \mathrm{~g}, 18.99 \mathrm{mmol})$ was added. A catalytic amount of sodium hydride $(\mu 5 \mathrm{mg})$ was then added to the solution, and the reaction mixture was stirred for $3 \mathrm{~h}$. The reaction was quenched with a half-saturated $\mathrm{NH}_{4} \mathrm{Cl}$ solution $(50 \mathrm{~mL})$. Upon separation of layers, the organic layer was washed with a saturated $\mathrm{NaHCO}_{3}$ solution $(50 \mathrm{~mL})$, which was then back-extracted with EtOAc $(3 \times 50 \mathrm{~mL})$. The organic layers were combined, dried over $\mathrm{MgSO}_{4}$, filtered, and concentrated in vacuo. The resulting crude material was purified with 80/20 hexanes/EtOAc to yield the Weinreb amide 21 in $57 \%$ yield $(2.39 \mathrm{~g}, 11.66 \mathrm{mmol})$ as a yellow oil. ${ }^{1} \mathrm{H}$ NMR $\left(400 \mathrm{MHz}, \mathrm{CDCl}_{3}\right): \delta(\mathrm{ppm}) 7.38$ $7.20(5 \mathrm{H}, \mathrm{m}), 6.51(1 \mathrm{H}, \mathrm{d}, J=15.9 \mathrm{~Hz}), 6.37(1 \mathrm{H}, \mathrm{ddd}, J=15.8,6.9,6.8 \mathrm{~Hz}), 3.72(3 \mathrm{H}, \mathrm{s})$, $3.39(2 \mathrm{H}, \mathrm{d}, J=6.4 \mathrm{~Hz}), 3.21(3 \mathrm{H}, \mathrm{s}) .{ }^{13} \mathrm{C} \mathrm{NMR}\left(100 \mathrm{MHz}, \mathrm{CDCl}_{3}\right): \delta(\mathrm{ppm}) 137.1,133.1$, 128.5, 127.4, 126.3, 122.8, 61.2, 36.5, 32.3 (b), 29.7. FT-IR $\left(\mathrm{cm}^{-1}\right): v 2936,1749,1722$, 1448, 1419, 1177, 999, 968, 910, 735. HRMS-ESI: $(\mathrm{M}+\mathrm{H})^{+} 206.1176$ calculated for $\mathrm{C}_{12} \mathrm{H}_{16} \mathrm{NO}_{2}$, experimental 206.1170.

The Weinreb amide $21(1.16 \mathrm{~g}, 5.65 \mathrm{mmol})$ was dissolved in dry THF $(50 \mathrm{~mL})$, and the

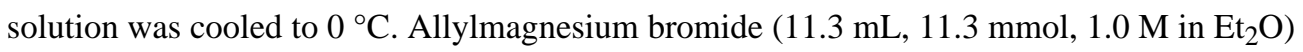
was then added slowly over $20 \mathrm{~min}$. The reaction mixture was stirred for $1 \mathrm{~h}$ and then quenched with a half-saturated $\mathrm{NH}_{4} \mathrm{Cl}(50 \mathrm{~mL})$ solution. Upon separation of layers, the aqueous layer was extracted with EtOAc $(3 \times 50 \mathrm{~mL})$. The organic layers were combined and dried over $\mathrm{MgSO}_{4}$. The crude material was concentrated in vacuo and purified with 90/10 hexanes/EtOAc to afford ketone 22 in $82 \%$ yield $(855 \mathrm{mg}, 4.59 \mathrm{mmol})$ as a yellow oil. ${ }^{1} \mathrm{H}$ NMR (400 MHz, $\left.\mathrm{CDCl}_{3}\right): \delta(\mathrm{ppm}) 7.38-7.22(5 \mathrm{H}, \mathrm{m}), 6.48(1 \mathrm{H}, \mathrm{d}, J=15.9 \mathrm{~Hz})$, $6.31(1 \mathrm{H}, \mathrm{ddd}, J=16.0,7.1,6.9 \mathrm{~Hz}), 6.00-5.89(1 \mathrm{H}, \mathrm{m}), 5.23-5.15(2 \mathrm{H}, \mathrm{m}), 3.36(2 \mathrm{H}, \mathrm{d}, J$ $=7.0 \mathrm{~Hz}), 3.27(2 \mathrm{H}, \mathrm{d}, J=6.9 \mathrm{~Hz}) .{ }^{13} \mathrm{C} \mathrm{NMR}\left(100 \mathrm{MHz}, \mathrm{CDCl}_{3}\right): \delta(\mathrm{ppm}) 206.4,136.8$, 133.9, 130.3, 128.6, 127.6, 126.3, 121.7, 119.2, 47.3, 46.4. FT-IR $\left(\mathrm{cm}^{-1}\right): v 3082,3061$, 3027, 2981, 1717, 1639, 1578, 1449, 1424, 1323, 1071, 993, 967, 912, 741, 695, 650. HRMS-ESI: $(\mathrm{M}+\mathrm{H})^{+} 187.1117$ calculated for $\mathrm{C}_{13} \mathrm{H}_{15} \mathrm{O}$, experimental 187.1117.

Ketone $22(855 \mathrm{mg}, 4.60 \mathrm{mmol})$ as a solution in $\mathrm{Et}_{2} \mathrm{O}(20 \mathrm{~mL})$ was added via cannula to a cooled $\left(0{ }^{\circ} \mathrm{C}\right)$ suspension of lithium aluminum hydride $(209 \mathrm{mg}, 5.50 \mathrm{mmol})$. The reaction mixture was then warmed to room temperature and set to reflux for $30 \mathrm{~min}$. After the reaction mixture was cooled to $0{ }^{\circ} \mathrm{C}$, deionized water $(0.21 \mathrm{~mL})$ was slowly added, which was followed by $15 \%$ aqueous sodium hydroxide solution $(0.21 \mathrm{~mL})$ and then deionized water $(0.63 \mathrm{~mL})$. This workup sequence resulted in the formation of white precipitates. The solution was then stirred for $1 \mathrm{~h}$. The filtrate was collected using vacuum filtration and concentrated in vacuo. The crude material was then purified with $90 / 10 \rightarrow 80 / 20$ hexanes/ EtOAc to give $8 \mathrm{f}$ with a yield of $94 \%\left(811 \mathrm{mg}, 4.31 \mathrm{mmol}\right.$ ) as a colorless oil. ${ }^{1} \mathrm{H}$ NMR (400 $\left.\mathrm{MHz}, \mathrm{CDCl}_{3}\right): \delta(\mathrm{ppm}) 7.37-7.20(5 \mathrm{H}, \mathrm{m}), 6.49(1 \mathrm{H}, \mathrm{d}, J=16.1 \mathrm{~Hz}), 6.25(1 \mathrm{H}, \mathrm{ddd}, J=$ 
15.9, 7.1, 6.9 Hz), $5.87(1 \mathrm{H}, \mathrm{m}), 5.19-5.14(2 \mathrm{H}, \mathrm{m}), 3.78(1 \mathrm{H}, \mathrm{m}), 2.48-2.33(3 \mathrm{H}, \mathrm{m}), 2.24$ $(1 \mathrm{H}, \mathrm{m}), 1.76(1 \mathrm{H}, \mathrm{m}) .{ }^{13} \mathrm{C}$ NMR $\left(100 \mathrm{MHz}, \mathrm{CDCl}_{3}\right): \delta(\mathrm{ppm}) 137.2,134.6,133.1,128.5$, 127.3, 126.1, 118.2, 70.2, 41.4, 40.5, 29.7. FT-IR $\left(\mathrm{cm}^{-1}\right): v 3416,3079,3027,2930,1641$, 1599, 1495, 1449, 1073, 997, 967, 912, 742. HRMS-ESI: $(\mathrm{M}+\mathrm{H})^{+} 189.1274$ calculated for $\mathrm{C}_{13} \mathrm{H}_{17} \mathrm{O}$, experimental 189.1275.

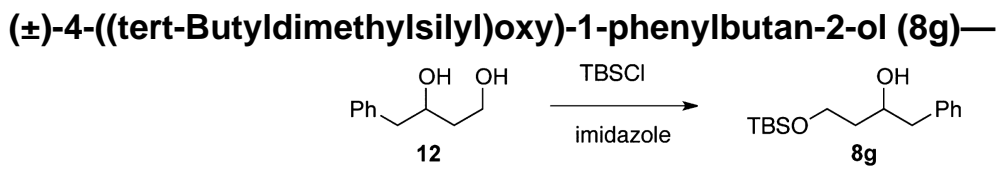

Diol $12{ }^{33}$ (479 mg, $2.88 \mathrm{mmol}$ ) was dissolved in $\mathrm{CH}_{2} \mathrm{Cl}_{2}$ (75 mL). Imidazole (432 mg, 6.34 mmol) was then added, and the reaction mixture was cooled to $-42{ }^{\circ} \mathrm{C}$. TBSCl $(478 \mathrm{mg}$, $3.17 \mathrm{mmol}$ ) was then added in one portion. The reaction mixture was stirred overnight while being slowly warmed to room temperature and then quenched with a half-saturated $\mathrm{NH}_{4} \mathrm{Cl}$ solution (50 mL). Upon separation of layers, the aqueous layer was extracted with $\mathrm{CH}_{2} \mathrm{Cl}_{2}$ $(3 \times 30 \mathrm{~mL})$, washed with brine, dried over $\mathrm{MgSO}_{4}$, filtered, and concentrated under vacuum. The crude material was purified with $90 / 10$ hexanes/EtOAc to give $8 \mathrm{~g}$ in $56 \%$ yield as a colorless oil (452 mg, $1.61 \mathrm{mmol}) .{ }^{1} \mathrm{H} \mathrm{NMR}\left(400 \mathrm{MHz}, \mathrm{CDCl}_{3}\right): \delta(\mathrm{ppm}) 7.32-7.20$ $(5 \mathrm{H}, \mathrm{m}), 4.08(1 \mathrm{H}, \mathrm{m}), 3.90(1 \mathrm{H}, \mathrm{p}, J=4.8 \mathrm{~Hz}), 3.79(1 \mathrm{H}, \mathrm{ddd}, J=10.4,6.5,6.3 \mathrm{~Hz}), 3.35$ $(1 \mathrm{H}, \mathrm{d}, J=2.0 \mathrm{~Hz}), 2.85(1 \mathrm{H}, \mathrm{dd}, J=13.5,7.0 \mathrm{~Hz}), 2.74(1 \mathrm{H}, \mathrm{dd}, J=13.5,6.2 \mathrm{~Hz}), 1.71-$ $1.66(2 \mathrm{H}, \mathrm{m}), 0.91(9 \mathrm{H}, \mathrm{s}), 0.08(6 \mathrm{H}, \mathrm{s}) .{ }^{13} \mathrm{C}$ NMR $\left(100 \mathrm{MHz}, \mathrm{CDCl}_{3}\right): \delta(\mathrm{ppm}) 138.8$, 129.4, 128.4, 126.3, 73.0, 62.6, 44.0, 37.6, 25.9, 18.2, -5.5. IR (cm-1): v3455, 2954, 2930, 2857, 1472, 1255, 1085, 909, 836, 777, 740, 700. HRMS-ESI: $(\mathrm{M}+\mathrm{H})^{+} 281.1931$ calculated for $\mathrm{C}_{16} \mathrm{H}_{29} \mathrm{O}_{2} \mathrm{Si}$, experimental 281.1922.

( \pm )-4-Benzyloxy-1-phenylbutan-2-ol (8h)-

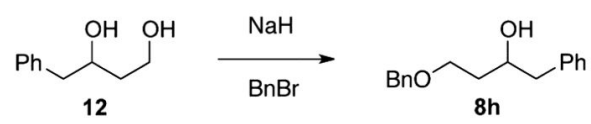

Diol $12{ }^{33}(2.78 \mathrm{~g}, 16.8 \mathrm{mmol})$ in a solution of DMF (20 mL) was slowly added via cannula to a cooled suspension $\left(0{ }^{\circ} \mathrm{C}\right)$ of $\mathrm{NaH}(0.426 \mathrm{~g}, 18.42 \mathrm{mmol})$ in DMF $(20 \mathrm{~mL})$. After the mixture was stirred for $30 \mathrm{~min}$, benzyl bromide $(1.99 \mathrm{~mL}, 16.8 \mathrm{mmol})$ was added. The reaction mixture was then warmed to room temperature and stirred overnight. After the reaction was quenched with a half-saturated $\mathrm{NH}_{4} \mathrm{Cl}$ solution, the mixture was then extracted with EtOAc $(3 \times 30 \mathrm{~mL})$, and the collected organic layers were washed with water and dried over $\mathrm{MgSO}_{4}$. The crude material was purified with 90/10 $\rightarrow 80 / 20 \rightarrow 70 / 30$ hexanes/ EtOAc, and alcohol $\mathbf{8 h}$ was isolated in $24 \%$ yield $(1.08 \mathrm{~g}, 3.95 \mathrm{mmol})$ as a yellow oil. ${ }^{1} \mathrm{H}$ NMR (400 MHz, CDCl $): \delta(\mathrm{ppm}) 7.36-7.19(10 \mathrm{H}, \mathrm{m}), 4.59(1 \mathrm{H}, \mathrm{d}, J=11.4 \mathrm{~Hz}), 4.48(1 \mathrm{H}$, d, $J=11.4 \mathrm{~Hz}), 3.86(1 \mathrm{H}, \mathrm{m}), 3.81-3.70(2 \mathrm{H}, \mathrm{m}), 3.04(1 \mathrm{H}, \mathrm{dd}, J=13.4,5.8 \mathrm{~Hz}), 2.79(1 \mathrm{H}$, $\mathrm{dd}, J=13.6,7.0 \mathrm{~Hz}), 2.22(1 \mathrm{H}, \mathrm{t}, J=5.0 \mathrm{~Hz}), 1.67-1.81(2 \mathrm{H}, \mathrm{m}) .{ }^{13} \mathrm{C} \mathrm{NMR}(100 \mathrm{MHz}$, $\left.\mathrm{CDCl}_{3}\right): \delta(\mathrm{ppm}) 138.4,138.1,129.5,128.5,128.4,128.0,127.8,126.3,79.6,71.6,60.7$, 40.5, 30.1. IR ( $\left.\mathrm{cm}^{-1}\right)$ : v3410, 3086, 3063, 3029, 2493, 2875, 1604, 1496, 1454, 1056, 1029, 910, 738, 699. HRMS-ESI: $(\mathrm{M}+\mathrm{H})^{+} 257.1536$ calculated for $\mathrm{C}_{17} \mathrm{H}_{21} \mathrm{O}_{2}$, experimental 257.1529 . 


\section{( \pm )-4-((4-Methoxybenzyl)oxy)-1-phenylbutan-2-ol (8i)-}

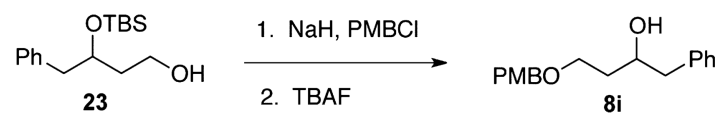

Alcohol $23^{9}(2.88 \mathrm{~g}, 10.3 \mathrm{mmol})$ was dissolved in THF $(150 \mathrm{~mL})$ and cooled to $0{ }^{\circ} \mathrm{C}$. NaH $(1.12 \mathrm{~g}, 46.8 \mathrm{mmol})$ was then added slowly, and the suspension was stirred for $30 \mathrm{~min}$. $\mathrm{PMBCl}(2.31 \mathrm{~g}, 14.8 \mathrm{mmol})$ was then added, and the reaction mixture was brought to room temperature and stirred overnight. The reaction was then slowly quenched with methanol $(60 \mathrm{~mL})$, followed by addition of a half-saturated brine solution $(50 \mathrm{~mL})$. Upon separation of layers, the aqueous solution was extracted with EtOAc $(3 \times 50 \mathrm{~mL})$. The organic layers were combined and washed with saturated $\mathrm{NaCl}$, dried over $\mathrm{MgSO}_{4}$, filtered, and concentrated under vacuum. The resulting crude material was dissolved in THF $(50 \mathrm{~mL})$ and treated with 1.0 M TBAF solution in THF $(20.5 \mathrm{~mL}, 20.56 \mathrm{mmol})$. After this solution was stirred overnight, EtOAc $(100 \mathrm{~mL})$ was added. This organic solution was washed sequentially with deionized $\mathrm{H}_{2} \mathrm{O}$ and brine, and it was then dried over $\mathrm{MgSO}_{4}$, filtered, and concentrated under vacuum. Purification of the resulting crude material with 80/20 $\rightarrow 70 / 30 \rightarrow 60 / 40$ hexanes/EtOAc gave alcohol $8 \mathrm{i}$ in $23 \%$ yield as a colorless oil $(664 \mathrm{mg}, 2.32 \mathrm{mmol}) .{ }^{1} \mathrm{H}$ NMR (400 MHz, $\left.\mathrm{CDCl}_{3}\right): \delta(\mathrm{ppm}) 7.32-7.20(7 \mathrm{H}, \mathrm{m}), 6.91-6.83(2 \mathrm{H}, \mathrm{m}), 4.52(1 \mathrm{H}, \mathrm{d}, J=$ $11.1 \mathrm{~Hz}), 4.41(1 \mathrm{H}, \mathrm{d}, J=11.0 \mathrm{~Hz}), 3.85(1 \mathrm{H}, \mathrm{m}), 3.81(3 \mathrm{H}, \mathrm{t}, J=4.2 \mathrm{~Hz}), 3.78-3.66(2 \mathrm{H}$, m), $3.03(1 \mathrm{H}, \mathrm{dd}, J=13.5,5.8 \mathrm{~Hz}), 2.77(1 \mathrm{H}, \mathrm{dd}, J=13.5,5.8 \mathrm{~Hz}), 1.81-1.62(2 \mathrm{H}, \mathrm{m}) .{ }^{13} \mathrm{C}$ NMR (100 MHz, $\left.\mathrm{CDCl}_{3}\right): \delta(\mathrm{ppm}) 159.3,138.4,130.2,129.6,128.7,128.4,126.3,113.9$, 79.4, 71.2, 60.7, 55.3, 40.5, 36.1. IR $\left(\mathrm{cm}^{-1}\right): v 3417,2941,1613,1514,1249,1035,822$, 741, 703. HRMS-ESI: $(\mathrm{M}+\mathrm{Na})^{+} 309.1467$ calculated for $\mathrm{C}_{18} \mathrm{H}_{22} \mathrm{NaO}_{3}$, experimental 309.1465

\section{(士)-3-Hydroxy-4-phenylbutyl 4-Methylbenzoate (8j)-}

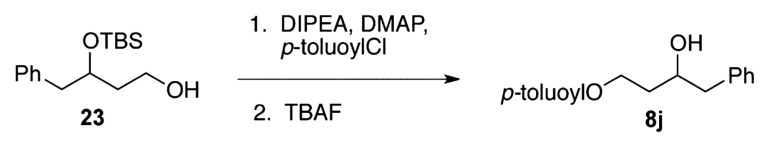

Alcohol $23{ }^{9}(1.12 \mathrm{~g}, 4.00 \mathrm{mmol})$ was dissolved in $\mathrm{CH}_{2} \mathrm{Cl}_{2}(40 \mathrm{~mL})$ and cooled to $0{ }^{\circ} \mathrm{C}$. DIPEA (3.56 mL, $20.0 \mathrm{mmol})$, DMAP $(0.24 \mathrm{~g}, 2.00 \mathrm{mmol})$, and $p$-toluoyl chloride $(0.63$ $\mathrm{mL}, 4.80 \mathrm{mmol}$ ) were sequentially added. After it was stirred for $3 \mathrm{~h}$, the reaction mixture was quenched with $2 \mathrm{M} \mathrm{HCl}$ solution $(40 \mathrm{~mL}$ ). Upon separation of layers, the aqueous solution was extracted with $\mathrm{CH}_{2} \mathrm{Cl}_{2}(3 \times 30 \mathrm{~mL})$, dried over $\mathrm{MgSO}_{4}$, filtered, and concentrated under vacuum. The resulting crude material was then dissolved in THF ( 25 $\mathrm{mL})$ and treated with 1.0 M TBAF solution in THF $(8.0 \mathrm{~mL}, 8.00 \mathrm{mmol})$. After the mixture was stirred overnight, EtOAc $(100 \mathrm{~mL})$ was added. This organic solution was washed sequentially with deionized $\mathrm{H}_{2} \mathrm{O}$ and brine, and it was then dried over $\mathrm{MgSO}_{4}$, filtered, and concentrated under vacuum. Purification of the resulting crude material with 80/20 hexanes/ EtOAc gave 8j in 65\% yield as a colorless oil (744 mg, $2.62 \mathrm{mmol}) .{ }^{1} \mathrm{H}$ NMR $(400 \mathrm{MHz}$, $\left.\mathrm{CDCl}_{3}\right): \delta(\mathrm{ppm}) 8.01-7.89(2 \mathrm{H}, \mathrm{m}), 7.34-7.23(7 \mathrm{H}, \mathrm{m}), 4.59(1 \mathrm{H}, \mathrm{ddd}, J=11.1,8.6,5.2$ $\mathrm{Hz}), 4.43(1 \mathrm{H}, \mathrm{p}, J=5.6 \mathrm{~Hz}), 4.01(1 \mathrm{H}, \mathrm{ddt}, J=8.6,8.6,4.3 \mathrm{~Hz}), 2.88(1 \mathrm{H}, \mathrm{dd}, J=13.6,4.6$ $\mathrm{Hz}), 2.78(1 \mathrm{H}, \mathrm{dd}, J=13.6,8.2 \mathrm{~Hz}), 2.44(1 \mathrm{H}, \mathrm{s}), 2.42(3 \mathrm{H}, \mathrm{s}), 2.03(1 \mathrm{H}, \mathrm{m}), 1.89(1 \mathrm{H}$, m). ${ }^{13} \mathrm{C} \mathrm{NMR}\left(400 \mathrm{MHz}, \mathrm{CDCl}_{3}\right): \delta(\mathrm{ppm})$ 167.0, 143.7, 138.1, 130.2, 129.7, 129.5, 129.1, 
128.6, 127.4, 126.6, 69.6, 62.0, 44.0, 36.0, 21.7. IR $\left(\mathrm{cm}^{-1}\right): v 3482,3029,2957,2919,1712$, 1612, 1455, 1274, 1178, 1111, 1021, 972, 842, 753, 701. HRMS-ESI: $(\mathrm{M}+\mathrm{H})^{+} 285.1491$ calculated for $\mathrm{C}_{18} \mathrm{H}_{21} \mathrm{O}_{3}$, experimental 285.1494.

\title{
Supplementary Material
}

Refer to Web version on PubMed Central for supplementary material.

\section{Acknowledgments}

\begin{abstract}
Generous financial support from Louisiana State University is greatly appreciated. A.V. thanks the National Science Foundation for the Bridge to the Doctorate Project (BDP) Fellowship (NSF1141152). C.E.A. thanks the Louisiana Board of Regents for the BOR Fellowship (LEQSF(2011-16)-GF-03). We thank Professor George Stanley for kindly allowing us to use the GC-MS instrument in his laboratories.
\end{abstract}

\section{ASSOCIATED CONTENT}

Supporting Information

Figures giving GC-MS chromatograms for Tables 1 and 2 and NMR $\left({ }^{1} \mathrm{H}\right.$ and $\left.{ }^{13} \mathrm{C}\right)$ spectra for characterized compounds. This material is available free of charge via the Internet at http://pubs.acs.org.

\section{REFERENCES}

(1). Cahiez G, Lefevre N, Poizat M, Moyeux A. Synthesis. 2013; 45:231.

(2). Denton RM, An J, Adeniran B, Blake AJ, Lewis W, Poulton AM. J. Org. Chem. 2011; 76:6749. [PubMed: 21744876]

(3). Braddock DC, Pouwer RH, Burton JW, Broadwith P. J. Org. Chem. 2009; 74:6042. [PubMed: 19591474]

(4). Shibuya GM, Kanady JS, Vanderwal CD. J. Am. Chem. Soc. 2008; 130:12514. [PubMed: 18717563]

(5). Yasuda M, Shimizu K, Yamasaki S, Baba A. Org. Biomol. Chem. 2008; 6:2790. [PubMed: 18633537]

(6). Dubey A, Upadhyay AK, Kumar P. Tetrahedron Lett. 2010; 51:744.

(7). Nilewski C, Carreira EM. Eur. J. Org. Chem. 2012:1685.

(8). Bedke DK, Vanderwal CD. Nat. Prod. Rep. 2011; 28:15. [PubMed: 21125121]

(9). Ayala CE, Villalpando A, Nguyen AL, McCandless GT, Kartika R. Org. Lett. 2012; 14:3676. [PubMed: 22780451]

(10). Eckert H, Forster B. Angew. Chem., Int. Ed. Engl. 1987; $26: 894$.

(11). Damle SB. Chem. Eng. News. 1993; 71:4.

(12). Cotarca L. Org. Process Res. Dev. 1999; 3:377.

(13). Lemoucheux L, Rouden J, Ibazizene M, Sobrio F, Lasne MC. J. Org. Chem. 2003; 68:7289. [PubMed: 12968878]

(14). Banwell MG, Coster MJ, Harvey MJ, Moraes JJ. Org. Chem. 2003; 68:613.

(15). Igarashi J, Kobayashi Y. Tetrahedron Lett. 2005; 46:6381.

(16). Roestamadji, J.; Mobashery, S.; Banerjee, A. Encyclopedia of Reagents for Organic Synthesis. Wiley; Hoboken, NJ: 2006.

(17). Kuna S, Pawlak Z, Tusk M. J. Chem. Soc., Faraday Trans. 1. 1982; 78:2685.

(18). Goren Z, Heeg MJ, Mobashery S. J. Org. Chem. 1991; 56:7186.

(19). Fu LQ, Liu X, Ling CY, Cheng JJ, Guo XS, He HL, Ding S, Yang YS. Bioorg. Med. Chem. Lett. 2012; 22:814. [PubMed: 22209487]

(20). Fang LJ, Yang JO, Yang F. Org. Lett. 2010; 12:3124. [PubMed: 20560543]

(21). Hemantha HP, Sureshbabu VV. Synlett. 2008:496. 
(22). Hajra S, Bhowmick M, Maji B, Sinha D. J. Org. Chem. 2007; 72:4872. [PubMed: 17542637]

(23). Masuda S, Nakajima T, Suga S. Bull. Chem. Soc. Jpn. 1983; 56:1086.

(24). Burk RM, Roof MB. Tetrahedron Lett. 1993; 34:395.

(25). Anderson EA, Davidson JEP, Harrison JR, O'Sullivan PT, Burton JW, Collins I, Holmes AB. Tetrahedron. 2002; 58:1943.

(26). Wolfe BH, Libby AH, Al-Awar RS, Foti CJ, Comins DL. J. Org. Chem. 2010; 75:8564. [PubMed: 21077636]

(27). Comins DL, Joseph SP, Goehring RR. J. Am. Chem. Soc. 1994; 116:4719.

(28). Sahn JJ, Bharathi P, Comins DL. Tetrahedron Lett. 2012; 53:1347.

(29). Kalyani D, Sanford MS. J. Am. Chem. Soc. 2008; 130:2150. [PubMed: 18229926]

(30). Kiyooka S, Shirouchi M. J. Org. Chem. 1992; 57:1.

(31). Canova S, Bellosta M, Mignani S, Bigot A, Cossy. J. Org. Lett. 2006; 8:2091.

(32). Pollastri MP, Sagal JF, Chang G. Tetrahedron Lett. 2001; 42:2459.

(33). Medlik-Balan A, Klein J. Tetrahedron. 1980; 36:299.

(34). Lin MH, Hung SF, Lin LZ, Tsai WS, Chuang TH. Org. Lett. 2011; 13:332. [PubMed: 21142089] 
<smiles>C[C@H](Cl)[C@H](Cl)[C@H](OS(=O)(=O)O)[C@H](Cl)[C@H](Cl)[C@H](Cl)CCCCCC/C=C/Cl</smiles>

Hexachlorosulfolipid<smiles>O=S(=O)(O)CC(Cl)(Cl)CCCCCCCC[C@H](Cl)C[C@H](Cl)[C@H](OS(=O)(=O)O)[C@H](Cl)[C@H](Cl)c1ccccc1</smiles>

Danicalipin A<smiles>CCCCCCCCCCCCCCCCCCCC(Cl)=COS(=O)(=O)O</smiles>

Figure 1.

Examples of chlorosulfolipid natural products. 


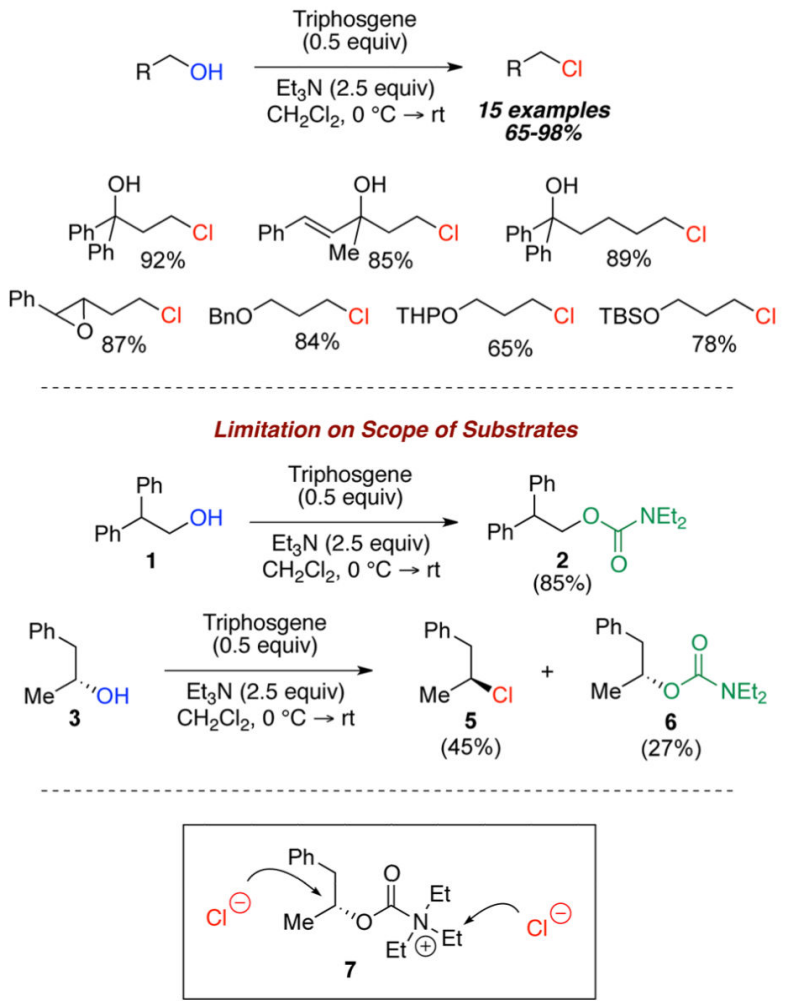

Scheme 1.

Previous Work on Triphosgene-Triethylamine-Promoted Chlorination 


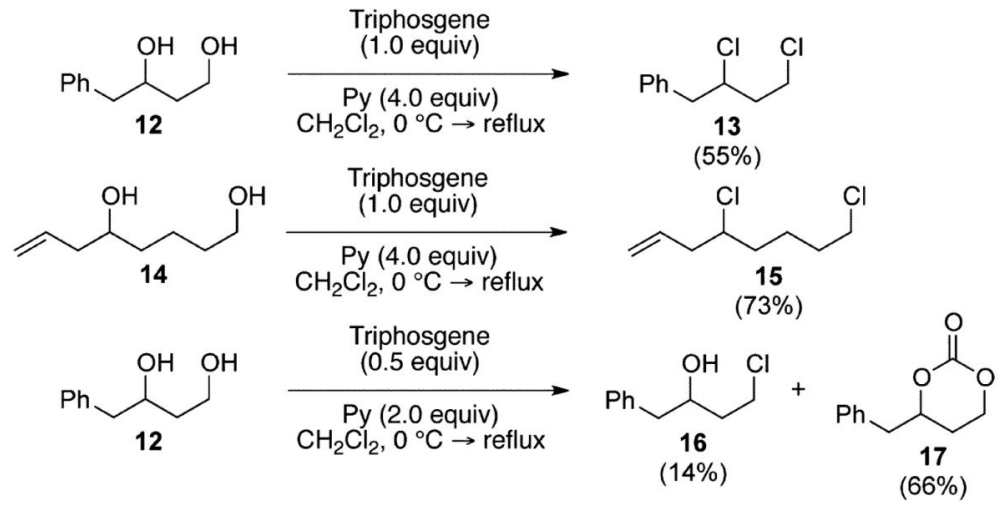

Scheme 2.

Reactivity of 1,3- and 1,6-Diols 

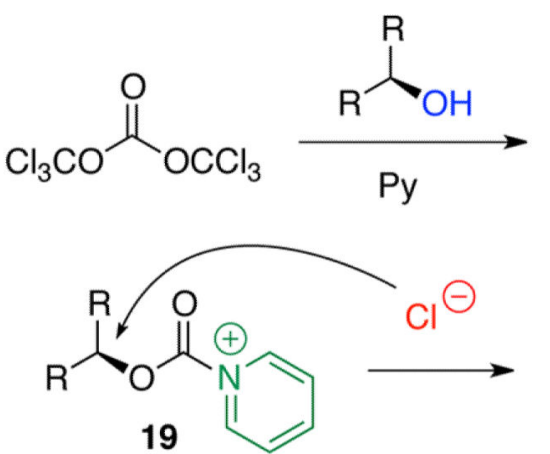

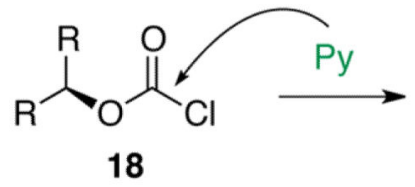

18

Scheme 3.

Proposed Reaction Mechanism 
Table 1

Optimization Study Varying the Amount of Triethylamine

\begin{tabular}{|c|c|c|c|c|c|c|}
\hline \multirow[b]{2}{*}{ entry } & \multirow[t]{2}{*}{ 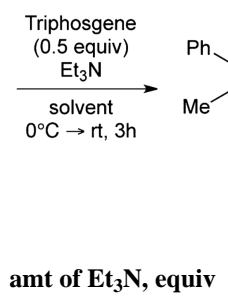 } & \multirow[b]{2}{*}{ solvent } & \multicolumn{3}{|c|}{ 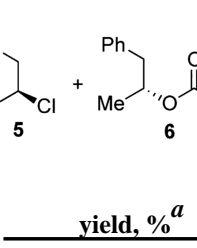 } & $\mathrm{NEt}_{2}$ \\
\hline & & & 3 & 4 & 5 & 6 \\
\hline 1 & 1.00 & $\mathrm{CH}_{2} \mathrm{Cl}_{2}$ & 6 & 73 & 14 & 7 \\
\hline 2 & 1.25 & $\mathrm{CH}_{2} \mathrm{Cl}_{2}$ & 3 & 75 & 13 & 9 \\
\hline 3 & 1.50 & $\mathrm{CH}_{2} \mathrm{Cl}_{2}$ & 2 & 45 & 27 & 27 \\
\hline 4 & 1.75 & $\mathrm{CH}_{2} \mathrm{Cl}_{2}$ & 1 & 16 & 44 & 39 \\
\hline 5 & 2.00 & $\mathrm{CH}_{2} \mathrm{Cl}_{2}$ & 0 & 3 & 51 & 46 \\
\hline 6 & 1.00 & toluene & 11 & 86 & 3 & 0 \\
\hline 7 & 1.25 & toluene & 3 & 87 & 5 & 5 \\
\hline 8 & 1.50 & toluene & 2 & 87 & 6 & 5 \\
\hline 9 & 1.75 & toluene & 1 & 54 & 18 & 27 \\
\hline 10 & 2.00 & toluene & 2 & 65 & 21 & 13 \\
\hline
\end{tabular}

${ }^{a}$ Yields were determined by GC-MS analysis of the crude mixtures, assuming that these compounds elicited identical GC responses. 
Table 2

Optimization Study with Mixed Amine Base Systems

\begin{tabular}{|c|c|c|c|c|c|c|c|}
\hline \multirow[b]{3}{*}{ entry } & \multirow{3}{*}{$\begin{array}{l}3_{3} \mathrm{OH} \\
\\
\text { equiv }\end{array}$} & $\begin{array}{c}\begin{array}{c}\text { Triphosgene } \\
(0.5 \text { equiv) } \\
\text { base }\end{array} \\
\underset{\text { conditions }}{\mathrm{CH}_{2} \mathrm{Cl}_{2}, 6 \mathrm{~h}}\end{array}$ & \multirow{3}{*}{$\begin{array}{rr}4 & 5 \\
& \\
\text { conditions }^{a} & \end{array}$} & \multirow{3}{*}{$\begin{array}{c}\mathrm{Ph} \\
\mathrm{Me}^{-} \\
\\
\end{array}$} & \multirow{2}{*}{\multicolumn{2}{|c|}{6}} & \multirow[b]{3}{*}{6} \\
\hline & & \multirow{2}{*}{$\begin{array}{c}\text { amt of } \\
\mathrm{Et}_{3} \mathrm{~N} \text {, equiv }\end{array}$} & & & & & \\
\hline & & & & & 4 & 5 & \\
\hline 1 & 1.2 & 0.25 & $0^{\circ} \mathrm{C} \rightarrow$ room temp & 0 & 98 & 1 & 1 \\
\hline 2 & 1.2 & 0.50 & $0^{\circ} \mathrm{C} \rightarrow$ room temp & 0 & 75 & 25 & 0 \\
\hline 3 & 1.2 & 0.75 & $0^{\circ} \mathrm{C} \rightarrow$ room temp & 0 & 60 & 39 & 0 \\
\hline 4 & 1.2 & 1.00 & $0^{\circ} \mathrm{C} \rightarrow$ room temp & 0 & 50 & 50 & 0 \\
\hline 5 & 1.2 & 0.25 & $0{ }^{\circ} \mathrm{C} \rightarrow$ reflux & 0 & 34 & 66 & 0 \\
\hline 6 & 1.2 & 0.50 & $0^{\circ} \mathrm{C} \rightarrow$ reflux & 0 & 9 & 90 & 1 \\
\hline 7 & 1.2 & 0.75 & $0^{\circ} \mathrm{C} \rightarrow$ reflux & 0 & 0 & 100 & 0 \\
\hline 8 & 1.2 & 1.00 & $0{ }^{\circ} \mathrm{C} \rightarrow$ reflux & 0 & 1 & 90 & 9 \\
\hline 9 & 1.0 & 0 & $0^{\circ} \mathrm{C} \rightarrow$ room temp & 93 & 0 & 7 & 0 \\
\hline 10 & 1.2 & 0 & $0^{\circ} \mathrm{C} \rightarrow$ room temp & 75 & 0 & 25 & 0 \\
\hline 11 & 1.7 & 0 & $0^{\circ} \mathrm{C} \rightarrow$ room temp & 35 & 0 & 65 & 0 \\
\hline 12 & 2.2 & 0 & $0^{\circ} \mathrm{C} \rightarrow$ room temp & 28 & 0 & 72 & 0 \\
\hline 13 & 1.0 & 0 & $0^{\circ} \mathrm{C} \rightarrow$ reflux & 69 & 0 & 31 & 0 \\
\hline 14 & 1.2 & 0 & $0^{\circ} \mathrm{C} \rightarrow$ reflux & 0 & 44 & 56 & 0 \\
\hline 15 & 1.7 & 0 & $0{ }^{\circ} \mathrm{C} \rightarrow$ reflux & 2 & 0 & 98 & 0 \\
\hline 16 & 2.2 & $\mathbf{0}$ & $0^{\circ} \mathbf{C} \rightarrow$ reflux & $\mathbf{0}$ & $\mathbf{0}$ & 100 & $\mathbf{0}$ \\
\hline
\end{tabular}

${ }^{a}$ Reagents were added at $0{ }^{\circ} \mathrm{C}$, and then the reaction mixture was warmed to room temperature or reflux.

${ }^{b}$ Yields were determined by GC-MS analysis of the crude mixtures, assuming that these compounds elicited identical GC responses. 
Table 3

Chlorination of Secondary Alcohols Containing Various Functionalities and Protecting Groups

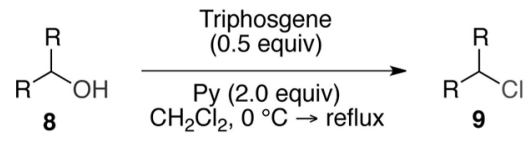

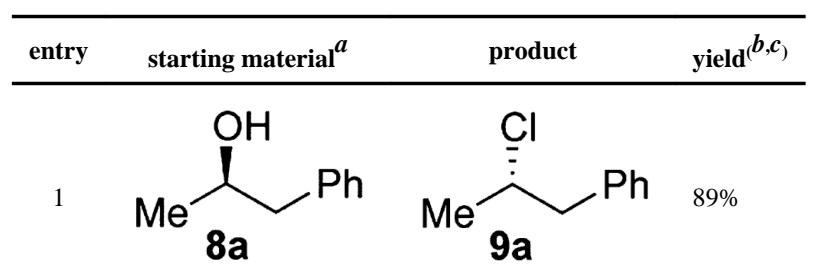

2<smiles>OC(Cc1ccccc1)Cc1ccccc1</smiles>

8b<smiles>ClC(Cc1ccccc1)c1ccccc1</smiles>

3<smiles>O=C1CN(C(=O)O)CCC1O</smiles><smiles>O=C(O)N1CCC(Cl)CC1</smiles>

4<smiles>CCOC(=O)CC(O)Cc1ccccc1</smiles><smiles>CCOC(=O)C(Cl)Cc1ccccc1</smiles>

5<smiles>C=CCC(O)Cc1ccccc1</smiles><smiles>C=CCC(Cl)Cc1ccccc1</smiles>

6<smiles>[CH]C(/C=C/c1ccccc1)C(O)CC=C</smiles><smiles>C=CCC(Cl)C(C)/C=C/c1ccccc1</smiles>

7<smiles>CCCCCCC(O)Cc1ccccc1</smiles><smiles>[18OH]CC(Cl)CC(Cl)c1ccccc1</smiles>

8<smiles>OC(CC(Cc1ccccc1)Cc1ccccc1)c1ccccc1</smiles><smiles>ClC(C[Hg]c1ccccc1)C(Cc1ccccc1)Cc1ccccc1</smiles>

9<smiles>CCCCC(C)CC(O)Cc1ccccc1</smiles><smiles>CCCCCC(Cl)CCc1ccccc1</smiles> 


\begin{tabular}{|c|c|c|c|}
\hline entry & starting material $^{a}$ & product & $\operatorname{yield}^{b, c}$ ) \\
\hline 10 & $p$-toluoylO & & $85 \%$ \\
\hline \multicolumn{4}{|c|}{${ }^{a}$ Alcohols $\mathbf{8 d - j}$ are racemic. } \\
\hline
\end{tabular}


Table 4

\section{Chlorination of $\alpha$-Branched Primary Alcohols}

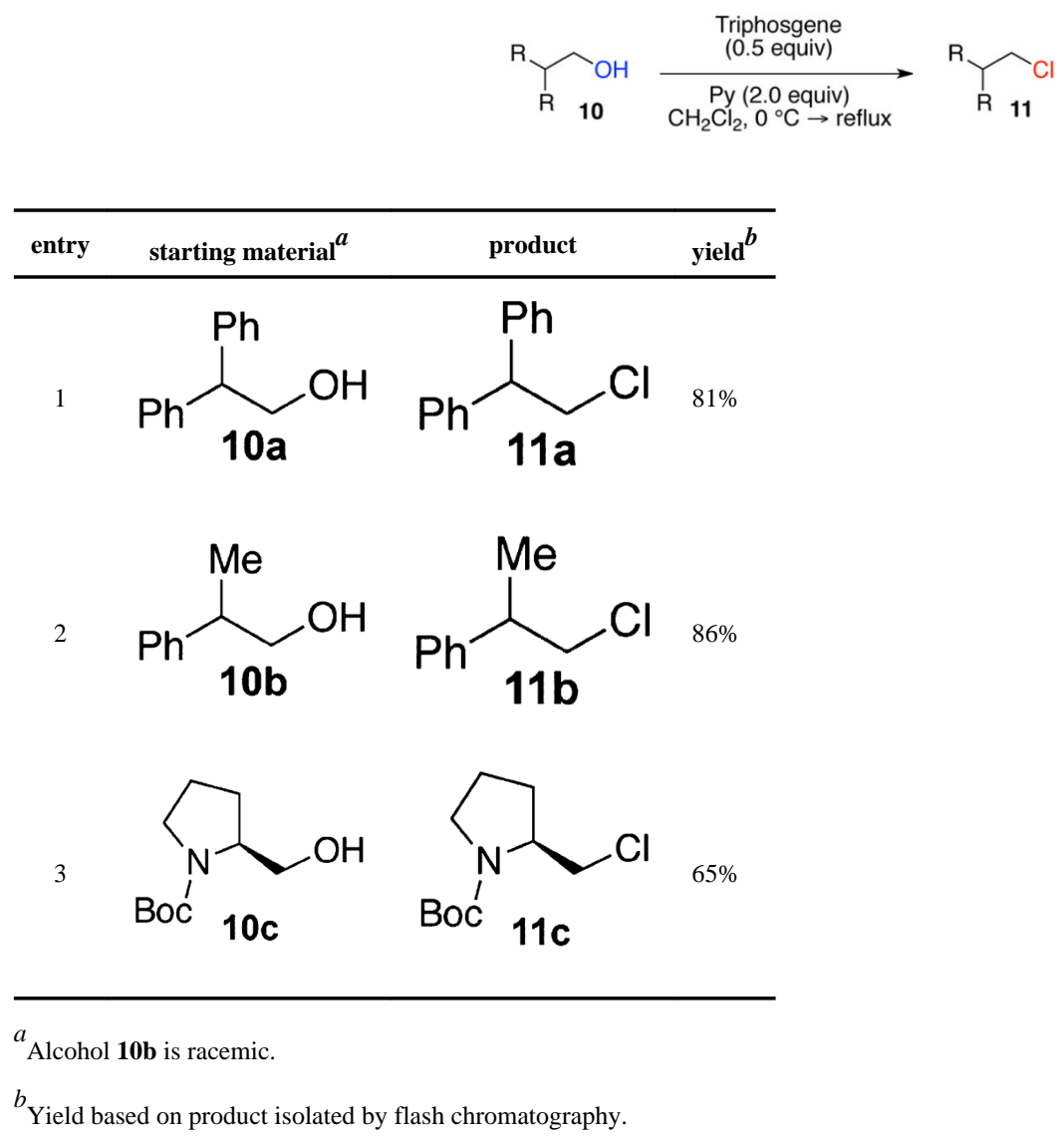

